\title{
Trichoderma atroviride LZ42 Releases Volatile Organic Com- pounds Promoting Plant Growth and Resisting Fusarium wilt Disease in Tomatoes
}

\author{
Hong Jiang ${ }^{1, \dagger}$, Yuxin Rao ${ }^{1} \uparrow$, , Linzhou Zeng ${ }^{1}$, Li Mei ${ }^{1}$, and Yongjun Wang ${ }^{1, *}$ \\ 1 Department of Forest Protection and Microbiology, College of Forestry and Biotechnology, Zhejiang A\&F \\ University, Hangzhou 311300, China; 610674352@qq.com (H.J.); 869642514@qq.com (Y.R.); \\ 2534281344@qq.com (L.Z.); meili@zafu.edu.cn (L.M.) \\ $\uparrow$ These authors equally contributed to the manuscript. \\ * Correspondence: wangyj@zafu.edu.cn ; Tel.: +86-57161067675
}

\begin{abstract}
The promotion of plant growth and suppression of plant disease using beneficial microorganisms is considered an alternative to the application of chemical fertilizers or pesticides in the field. In this study, a coconut-scented antagonistic Trichoderma strain LZ42, previously isolated from Genoderma lucidum-cultivated soil, was investigated for biostimulatory and biocontrol functions in tomato seedlings. Morphological and phylogenetic analyses suggested that strain LZ42 is closely related to T. atroviride. Tomato plants showed increased aerial and root dry weights in greenhouse trials after treatment with T. atroviride LZ42 formulated in talc, indicating the biostimulatory function of this fungus. T. atroviride LZ42 effectively suppressed Fusarium wilt disease in tomato seedlings, with an $82.69 \%$ control efficiency, which is similar to that of fungicide treatment. The volatile organic compounds (VOCs) emitted by T. atroviride LZ42 were found to affect the primary root growth direction and promote the root growth of tomato seedlings in root Y-tube olfactometer assays. The fungal VOCs from T. atroviride LZ42 were observed to significantly inhibit $F$. oxysporum in a sandwiched Petri dish assay. SPME-GC-MS analysis revealed several VOCs emitted by $T$. atroviride LZ42; the dominant compound was tentatively identified as 6-pentyl-2H-pyran-2-one (6-PP). 6-PP exhibited a stronger ability to influence the direction of the primary roots of tomato seedlings but not the length of the primary roots. The inhibitory effect of 6-PP on F. oxysporum was the highest among the tested pure VOCs, showing a 50\% effective concentration (EC50) of $5.76 \mu \mathrm{L} \mathrm{mL} \mathrm{m}^{-1}$ headspace. In conclusion, T. atroviride LZ42, which emits VOCs with multiple functions, is a promising agent for the biostimulation of vegetable plants and integrated management of Fusarium wilt disease.
\end{abstract}

Keywords: Trichoderma atroviride; volatile organic compounds; tomato; biostimulation; biocontrol; Fusarium wilt disease

\section{Introduction}

Members of Trichoderma spp. are ubiquitous in the soil and wood as soil inhabitants, plant symbionts, and mycoparasites [1]. Certain species of this genus are the most versatile biofertilizer and/or biocontrol agents and are presently used as active ingredients in biopesticides, biofertilizers, growth enhancers, and stimulants of natural resistance [2,3]. Over $60 \%$ of all the biopesticides registered in China contain a single Trichoderma isolate or mixtures of Trichoderma species (http://www.chinapesticide.org.cn/ywb/index.jhtml, August 4, 2021). The well-known species that have been commercially developed include T. harzianum, T. virens, T. viride, T. asperellum, and T. atroviride [4]. These fungi are considered safe for human and livestock, and beneficial for crop plants through colonizing plant roots, without apparent adverse responses [5]. 
In recent decades, the multiple biological functions of Trichoderma, including the suppression of plant disease, the promotion of plant growth, decomposition, and bioremediation, have been well-documented. Trichoderma spp. have been reported to exhibit antagonistic activity against a broad spectrum of plant pathogenic fungi and oomycetes, such as Fusarium oxysporum [6,7] and Phytophthora colocasiae [8], as well as plant pathogenic bacteria [9] and nematodes [10]. The many secondary metabolites produced by Trichoderma species are responsible for their highly antagonistic effects, contributing to the success of their application [11]. Additionally, the application of Trichoderma species successfully regulates root architecture, increasing the length of lateral and primary roots, leading to the more efficient uptake of nutrients by the plant, resulting in biostimulation [12-14]. The mechanism through which Trichoderma exerts biostimulatory effects involves multilevel communication with root and shoot systems, including releasing many active metabolites into the rhizosphere, thereby increasing plant growth and yield [2].

Among the diverse secondary metabolites produced by Trichoderma species, a group of gas-phase, carbon-based compounds that are able to diffuse through the atmosphere and soils, called volatile organic compounds (VOCs), exhibit multiple biological functions [15-17]. The VOCs detected for Trichoderma comprise simple hydrocarbons, heterocycles, aldehydes, ketones, alcohols, phenols, thioalcohols, thioesters, and their derivatives [18]. Over 500 different VOCs in Trichoderma species had been detected according to a database of microbial volatiles (mVOC database) [19]. Some of these VOCs were reported to induce plant resistance or directly promote plant growth [20-22], and some were shown to be detrimental to plant pathogens $[23,24]$. These findings indicate that VOCs may play a role in the biostimulatory and biocontrol activities of Trichoderma spp. $[12,16,25]$.

Our previous study demonstrated the abundance of antagonistic Trichoderma species growing on the continuously cultivated soil surface of Ganoderma lucidum, an important Chinese traditional medicinal mushroom with high economic value [26]. Regardless of the continuous cultivation of the mushroom leading to problems due to increased populations of Trichoderma, the diverse species of Trichoderma in mushroom houses might provide bioresources for biostimulation and biocontrol in vegetable crop production. In this study, three Trichoderma strains with strong coconut-like scents were identified and investigated as potential biocontrol agents and plant stimulants. In addition, the emitted VOCs were analyzed; the antagonistic activity and growth-promoting activity of the VOCs were determined.

\section{Materials and Methods}

\subsection{Trichoderma and plant pathogens}

The antagonistic Trichoderma strain LZ42 was previously isolated from G. lucidum cultivated soil in Zhejiang province in China [26]. The plant pathogen Fusarium oxysporum f. sp. lycopersici K34 used in this study was obtained from the National and Provincial Joint Engineering Laboratory of Biopesticide Preparation, Zhejiang A\&F University, China. All the fungi were grown on potato dextrose agar (PDA) medium at $26{ }^{\circ} \mathrm{C}$ and restreaked after one month.

\subsection{Plant material and growth conditions}

Tomato seeds (ZheZa 806 ) were surface-sterilized with $2 \%$ sodium hypochlorite $(\mathrm{NaOCl})$ for $5 \mathrm{~min}$, rinsed four times for $5 \mathrm{~min}$ in deionized sterilized water, and sown in foam pots containing a mixture of perlite, peat, and vermiculite $(1: 1: 1 \mathrm{v} / \mathrm{v} / \mathrm{v})$. The plants were grown at a maximum temperature of $30^{\circ} \mathrm{C}$ (day) and minimum of $21^{\circ} \mathrm{C}$ (night) at a relative humidity of $60-85 \%$ under daylight conditions in a greenhouse.

\subsection{Morphological observation}

The growth rates were determined on $9 \mathrm{~cm}$-diameter Petri dishes containing PDA and cornmeal dextrose agar (CMD, Oxoid, Hants, U.K.). The morphological characteris- 
tics of the colonies were recorded. Microscopic morphological observations were performed with a Motic M200 microscope (Motic, China) and a scanning electron microscope (Phenom Pro, the Netherlands). The fungus grew on $\mathrm{CMD}$ at $25{ }^{\circ} \mathrm{C}$. Conidia were investigated at 14 days after incubation.

\subsection{Molecular identification}

The DNA of strain LZ42 was extracted using the Ezup Column Fungi Genomic DNA Purification Kit (Sangon Biotech, Shanghai, China) according to the manufacturer's protocol. DNA fragments of RNA polymerase II subunit B (rpb2) and translation elongation factor 1-alpha (tef1) were amplified with the primer pairs fRPB2-5f/fRPB2-7cr [27] and EF1-728F/ TEF1LLErev [28], respectively. These obtained sequences were deposited in the GenBank database at the National Center for Biotechnology Information (NCBI), and the accession numbers are listed in Supplementary Table S1. The combined sequence of $r p b 2$ and tef1 from strain LZ42 was assessed by phylogenetic analysis for its variability compared to other species of Trichoderma in the NCBI using Mega 7.0 [29]. The phylogeny was analyzed by the bootstrap method with 1000 replications and condensed with a cut-off value of $70 \%$.

\subsection{Talc formulation of strain $L Z 42$}

Mycelial discs $(9 \mathrm{~mm})$ of strain LZ42 grown on PDA medium were inoculated in $1000 \mathrm{~mL}$ of PD broth and incubated at room temperature for five days. Afterward, the fungal culture was blended thoroughly and mixed with talc powder (XuFeng@, Quanzhou, China) at a ratio of 1:2 (v/w). The mixture was air-dried and ground gently. Subsequently, the formulated strain LZ42 was assessed for its concentration in colony-forming units (CFU) in PDA medium and had a minimum count of $2.0 \times 10^{7} \mathrm{CFU} / \mathrm{g}$.

\subsection{Greenhouse assessment}

The tomato seedlings were gently transplanted to pots containing $1 \mathrm{~kg}$ of soil or Fusarium-infested soil after 30 days of sowing for further experiments. Fusarium-infested soil was obtained by mixing a F. oxysporum spore suspension as described previously [30]. Seven treatments were applied as follows: (1) NC, uninfested soil; (2) FI, Fusarium-infested soil; (3) MT, uninfested soil amended with $50 \mathrm{~g}$ of the strain LZ42 formulation; (4) MC, uninfested soil amended with $50 \mathrm{~g}$ of the talc formulation alone; (5) FT, Fusarium-infested soil amended with $50 \mathrm{~g}$ of the strain LZ42 formulation; (6) FA, Fusarium-infested soil amended with $50 \mathrm{~g}$ of the talc formulation alone; (7) FC, Fusarium-infested soil mixed with carbendazim, $10 \mathrm{~mL}$ of $2.0 \mathrm{mg} \mathrm{mL}^{-1}$ carbendazim solution (25\%, wettable powder, dissolved in water). Ten pots were used in each treatment. Each experiment was performed independently 3 times. The wilt incidences for tomato seedlings were calculated based on the wilt symptoms at 50 days post-inoculation (dpi). The disease incidence is reported as the percentage of diseased plants out of the total number of plants. For the determination of the dry plant weight, the plants were thoroughly washed with water and dried at $70{ }^{\circ} \mathrm{C}$ until reaching a constant weight.

\subsection{Root Y-tube olfactometer assay}

As described by Moisan et al. (2021) [31], a Y-tube olfactometer consisting of a Y-shaped plastic tube (Yinzhan, Hangzhou, China) and two arms was used to study the volatile-mediated interactions between Trichoderma and plants. We applied some minor modifications for growing tomato seedlings in the olfactometers. The Y-shaped plastic tube was filled with a mixture of perlite, peat, and vermiculite $(1: 1: 1, \mathrm{v} / \mathrm{v} / \mathrm{v})$ with the two arms pointing downward. Each arm was connected to an empty tube that was connected to a vial containing agar medium with or without Trichoderma. After assembling all the tubes together, sterile seeds were sown on the soil surface on top of the Y-tubes. The primary roots of the germinated tomato seedlings could grow downward into one of the two arms of the Y-tube: either toward the treatment or toward the control. One seed was sown per olfactometer, and 30 independent root olfactometers were used in each treatment. The assembled root olfactometers were buried in a plant nursery bed filled with sand and placed in a greenhouse. Plants were harvested 20 days after sowing. The 
"choice" of the primary roots was calculated. The roots were collected and washed to remove soil particles. The lengths of the primary roots were measured, and all the roots were dried at $70{ }^{\circ} \mathrm{C}$ and weighed.

\subsection{Antagonistic activity assay for volatiles produced by strain LZ42}

Sandwiched Petri plates, a setup described by Li et al. (2018) [24], were employed to test the antagonistic activity of the volatiles produced by strain LZ42 against the plant pathogen F. oxysporum. After inoculating the pathogen and strain LZ42 on PDA plates, the pathogen plate was placed on top of a Trichoderma plate, sealed with three layers of parafilm, and then incubated at $25^{\circ} \mathrm{C}$. A non-inoculated PDA plate was set as the control. The colony diameters were measured 5 days later. Each treatment included three biological replicates and was repeated three times.

\subsection{Analysis of the contents of the volatiles produced by strain LZ42}

Qualitative assays of the composition of the volatiles produced by strain LZ42 were performed using solid-phase microextraction coupled with gas chromatography tandem mass spectrometry (SPME-GC-MS) analysis [32]. Strain LZ42 was cultured on PDA and incubated at $28^{\circ} \mathrm{C}$ for 5 days. SPME inserted into the injection port of a GC 2010 gas chromatograph was used to collect the volatiles according to the method previously described by $\mathrm{Wu}$ et al. (2020) [33]. SPME fiber was exposed to the vapor phase above strain LZ42 for $45 \mathrm{~min}$ in a culture tube. Then, the adsorbent fiber was inserted into the injection port of a Hewlett Packard 7890GC/5975MSD gas chromatograph (Agilent Technologies, USA) equipped with an HP-5MS capillary column. Purified helium was used as the carrier gas with a flow rate of $1 \mathrm{~mL} / \mathrm{min}$ in split-splitless mode. The column temperature was programmed as follows: an initial temperature of $40{ }^{\circ} \mathrm{C}$ for $2 \mathrm{~min}$, increased at a rate of $4{ }^{\circ} \mathrm{C}$ min- 1 to $180{ }^{\circ} \mathrm{C}$, and then increased to $250{ }^{\circ} \mathrm{C}$ at a rate of $5{ }^{\circ} \mathrm{C} \mathrm{min}-1$, before being maintained at $250{ }^{\circ} \mathrm{C}$ for $6 \mathrm{~min}$. The mass spectrometer was operated at unit mass resolution. The derived data were analyzed and identified based on a comparison with the mass spectrum of the GC-MS system in the NIST08.L data bank (National Institute of Standards and Technology). Each experiment was conducted three times.

\subsection{Antifungal activity assay for selected pure VOCs}

The antifungal activity of each selected pure VOC was tested by fumigation in an isolated container as described in Wu et al. (2020) [33]. Mycelial plugs of F. oxysporum (5 $\mathrm{mm}$ in diameter) were placed in the center of the PDA. Filter paper (30 $\mathrm{mm}$ in diameter) with $1,2,4,8,16$, or $32 \mu \mathrm{L}$ of each pure VOC (Sigma-Aldrich, MO, USA) was attached inside the cover of the dishes, resulting in $0.01,0.03,0.05,0.11,0.21$, and $0.43 \mu \mathrm{L} \mathrm{mL}^{-1}$ headspace, respectively. Subsequently, the loaded plates were immediately sealed with three layers of parafilm and incubated at $25^{\circ} \mathrm{C}$. The assays for each dose of pure VOCs were conducted with three replicates. Plates with filter paper alone were used as the controls. The percentage inhibition of mycelial growth was calculated according to the formula $(c-t / c) \times 100$, where $t$ is the mean diameter of the fungal colonies following the treatment and $c$ is the mean value for the control. The EC 50 values (expressed as $\mu \mathrm{L} \mathrm{mL}^{-1}$ headspace) were calculated as the effective concentration that inhibited mycelial growth by $50 \%$ in comparison to the control.

\subsection{Statistical Analysis}

Tukey's HSD test was used to assess the differences between means for the antagonistic activity and growth promotion of tomatoes for treatment with T. atroviride LZ42 or the fungal VOCs. The "choice" of the primary roots was analyzed with binomial tests using a $\chi 2$ test $\left(\mathrm{H}_{0}=0.50\right)$. Differences in plant dry weights between the control and treatment were analyzed with Student $t$-tests. The significance was evaluated at $\mathrm{P}=0.05$ in all the tests. The statistical analyses were performed in the IBM SPSS Statistics v.19 program (SPSS, Inc.).

\section{Results}

3.1. Identification and morphological characterization of the scented strain LZ42 
During the isolation and culture of the Trichoderma isolates from soil under continuous G. lucidum cultivation in China, three of the total of 214 isolates emitted a strong coconut-like scent when fungi grew on PDA plates 5 days after inoculation. These three isolates exhibited the same ITS sequences and the same colony morphological characteristics (data not shown). Isolate LZ42 was selected for further identification and research as the representative strain. The characterization of the combinations of $\mathrm{rdp} 2$ and tef1 revealed the identity of strain LZ42 to be T. atroviride. Phylogenetic analysis based on the combinations of the rdp2 and tef1 gene sequences of different Trichoderma spp. revealed strain LZ42 to be more closely related to T. atroviride in the neighbor joining tree (Figure 1A). Strain LZ42 grown on a PDA plate for 3 days exhibited a well-defined hyaline colony with typical aerial hyphae (Figure 1B). Strong coconut-like odors were released when the plate was opened. The fungal conidiation started after 4 days, followed by a change from white to dark green in the outer half of the colony (Figure 1C). The conidiophores emerged radially from the slender reticulum, consisting of a main axis and often distantly spaced side branches (Figure 1D). The phialides formed on a widened supporting cell at the end of the conidiophores. The conidia were sub-globose to globose, smooth, and thick-walled (Figure 1E).
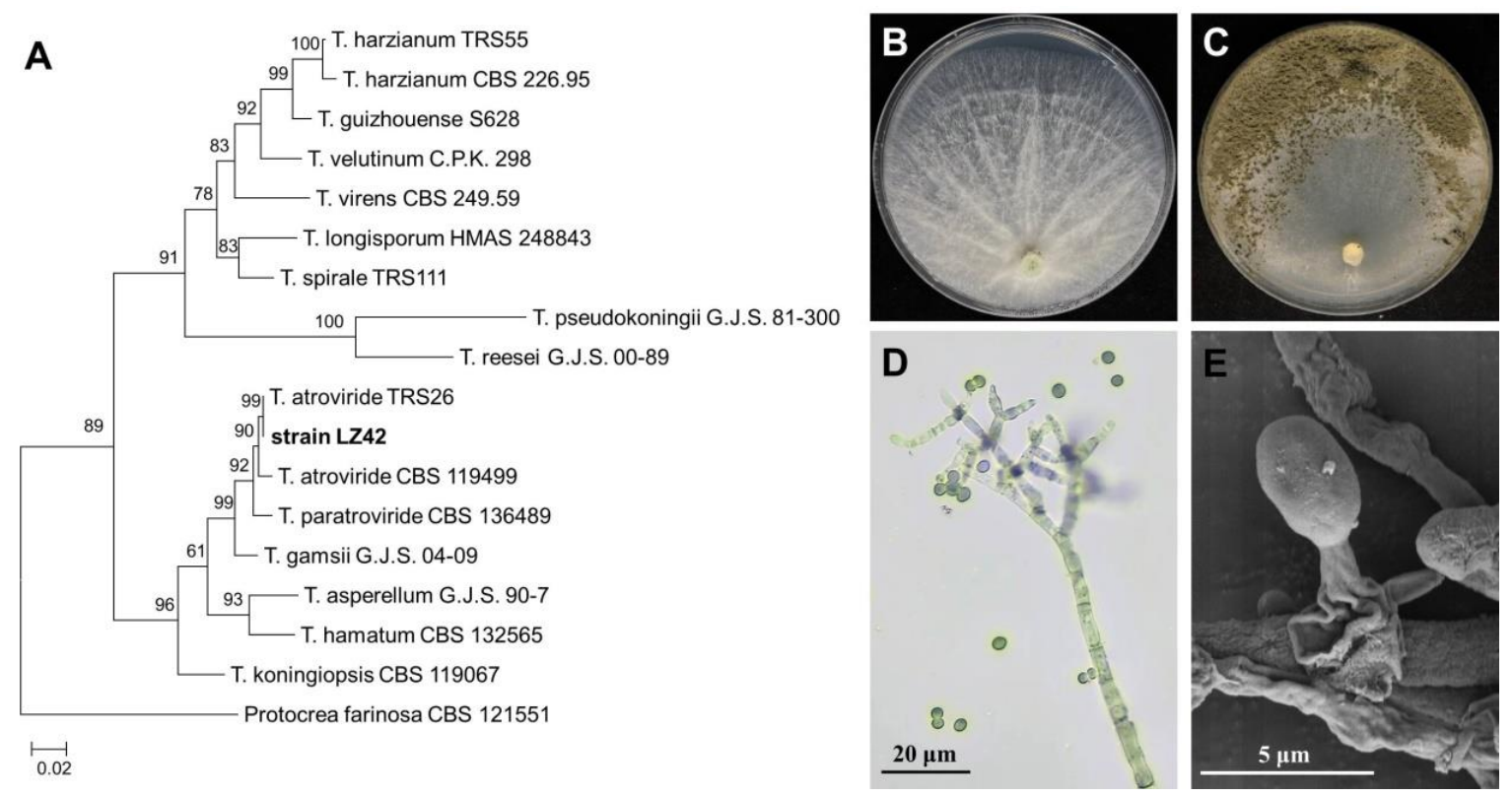

Figure 1. Molecular identification and morphological characteristics of Trichoderma atroviride LZ42. (A) Phylogenetic tree generated from the combined sequences of $r p b 2$ and tef1 loci of the genus Trichoderma spp. The tree was formulated using Mega 7.0 with 1000 bootstrap replications, condensed with a 70\% cut-off value. (B) Colony of T. atroviride LZ42 grown for 7 days on PDA. (C) Colony of

T. atroviride LZ42 grown for 14 days on CMD. (D) Conidiophores and conidia formed on CMD under optical microscopy. (E)

Conidiophores and conidia formed on CMD under SEM.

3.2. The effect of Trichoderma atroviride LZ42 formulation on the plant vegetable growth and control of Fusarium wilt disease

At 15 days post-inoculation with T. atroviride LZ42 formulated in talc, the plant growth of the tomatoes was significantly promoted, as reflected in the increases in the aerial and root dry weights of the total tomato plants (Fig. 2). The mean aerial and root dry weights of the tomatoes treated with T. atroviride LZ42 were $0.74 \mathrm{~g}$ and $0.21 \mathrm{~g}$, respectively, which were increases of $37.03 \%$ and $31.25 \%$ compared to the those for the treatment with buckwheat alone, respectively. We observed no differences between the negative control and treatment with talc alone. 

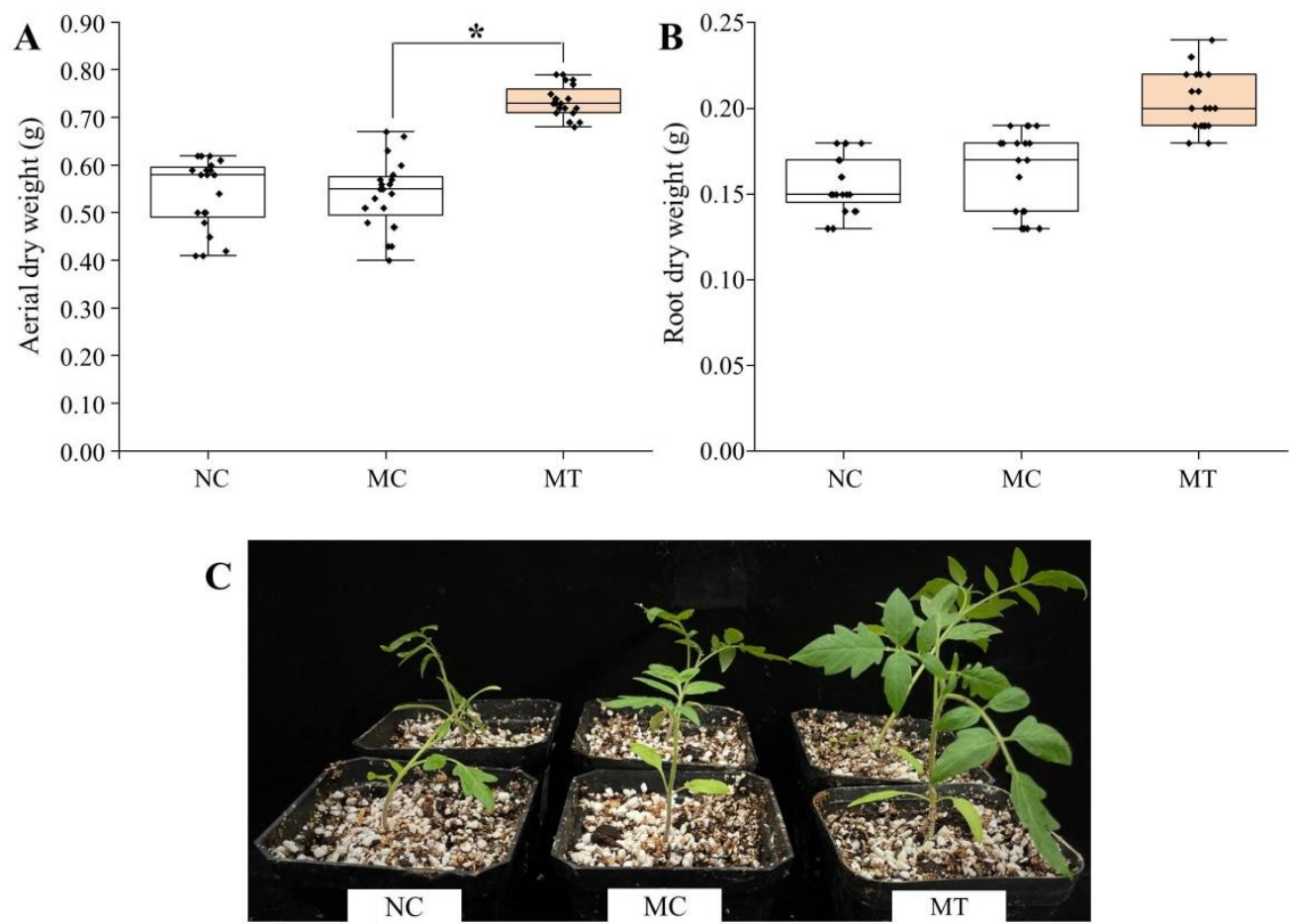

Figure 2. Effects of Trichoderma atroviride LZ42 on the phenotypic characteristics of aerial dry weight and root dry weight of tomatoes. Asterisks $\left(^{*}\right)$ in the figure indicate significant differences derived from two sample comparisons $(P=0.05)$. The NC, MC, and MT treatments are described in the Materials and Methods.

The incidence of tomato Fusarium wilt disease after $15 \mathrm{dpi}$ is summarized in Table 1. The fungal pathogen F. oxysporum K34 exhibited high virulence to tomato seedlings in the greenhouse, with a $59.67 \%$ disease incidence. We found significant $(\mathrm{P}=0.05)$ differences between the FT $(10.25 \%)$ and FI treatments $(59.67 \%)$ in disease incidence. The control efficiency of the T. atroviride LZ42 formulation was $82.69 \%$. No significant differences between the FP and FC treatments were observed, indicating that T. atroviride LZ42 reduced the disease incidence with an efficiency equal to that of carbendazim.

Table 1. Efficacy of Trichoderma atroviride strain LZ42 and carbendazim in control of Fusarium wilt on tomato seedlings.

\begin{tabular}{ccc}
\hline Treatment & \% Disease incidence & \% Control efficiency \\
\hline FA & $59.67 \pm 3.21 \mathrm{~b}$ & - \\
FT & $10.33 \pm 1.53 \mathrm{a}$ & 82.69 \\
FC & $7.67 \pm 1.15 \mathrm{a}$ & 87.15 \\
\hline
\end{tabular}

FA, FT, and FC treatments are described in the Materials and Methods. Values are means of 10 replicates \pm standard errors (SEs). Different superscripted uppercase letters indicate values that are significantly different $(\mathrm{P}=0.05)$ within the same columns.

3.3. Effects of VOCs produced by Trichoderma atroviride LZ42 on root growth of tomato seedlings in $Y$-tube olfactometers

The effects of the volatiles produced by T. atroviride LZ42 on the directional growth and growth rate of tomato seedling roots were tested in Y-tube olfactometers (Fig. 3A). Primary roots grew more frequently in the arm toward volatiles from T. atroviride LZ42 than in the arm toward volatiles from the agar medium control (Figure 3B; Obs.Freq = $0.63 ; \mathrm{P}=0.125)$. The means of the length of the primary roots and total root dry weight with treatment with VOCs from T. atroviride LZ42 were $17.31 \mathrm{~cm}$ and $5.88 \mathrm{mg}$, respectively, which are higher than those of the control $(13.65 \mathrm{~cm}$ and $4.67 \mathrm{mg}$, respectively; $\mathrm{P}=0.05$; Figure $3 \mathrm{C}$ and Figure 3D). 

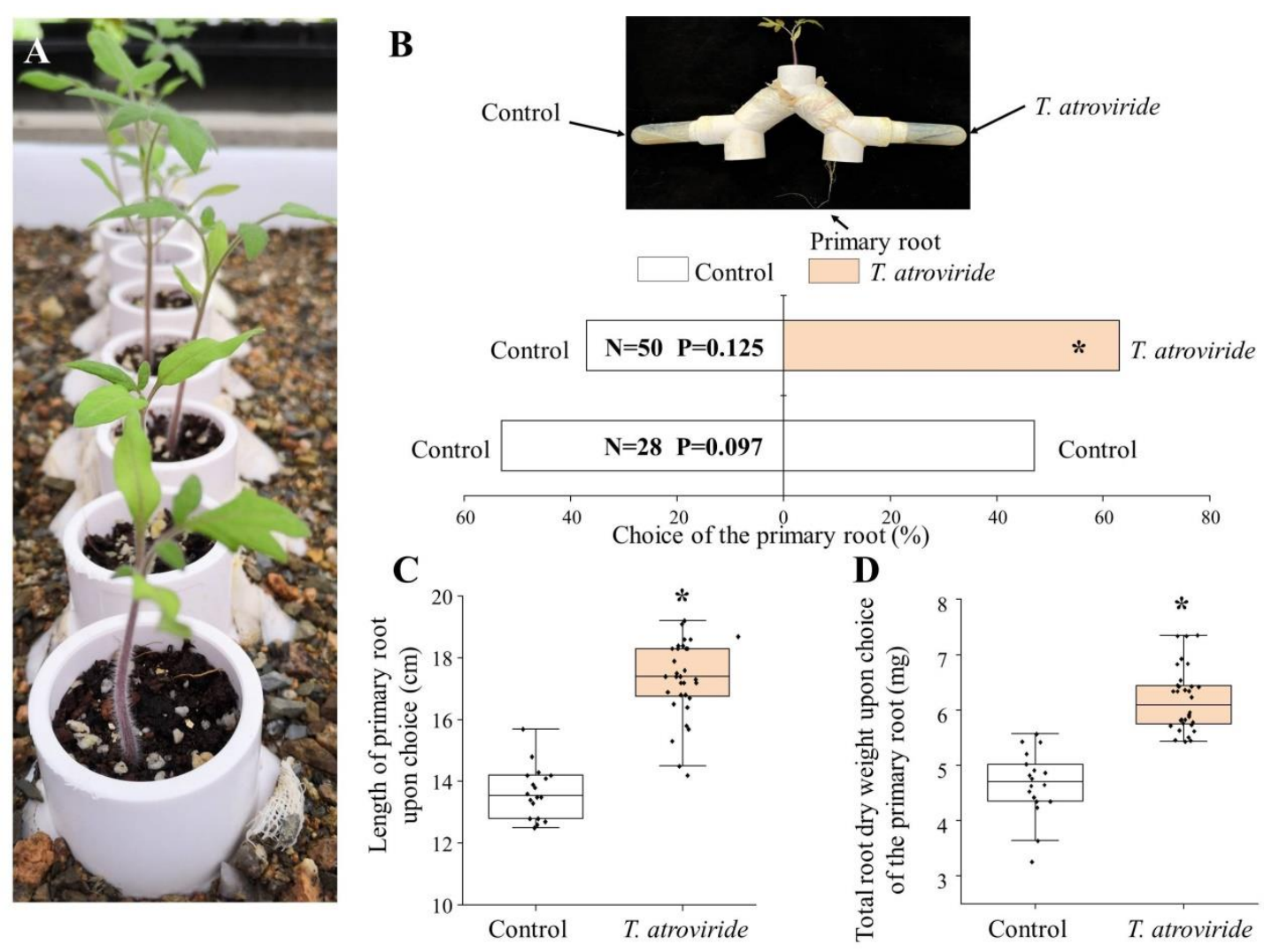

Figure 3. Effects of the VOCs produced by Trichoderma atroviride LZ42 on the root growth of tomato seedlings in modified root Y-tube olfactometers. (A) Tomato seedlings grown in the root Y-tube olfactometers for 14 days. (B) Percentages of tomato primary roots growing in the control arm or the T. atroviride arm 14 days after sowing. Control versus control was used as a negative control. $\mathrm{N}$, the number of replicates. Asterisks, pairwise differences between the control and T. atroviride choices $\left(\mathrm{H}_{0}=0.50\right)$. $(\mathrm{C})$ Length of the primary root. (D) Total root dry weight of the primary root. Asterisks $\left.{ }^{*}\right)$ in the figure indicate significant differences derived from two sample comparisons $(\mathrm{P}=0.05)$.

\subsection{Antifungal activity of VOCs produced by Trichoderma atroviride LZ42}

To determine the effects of the VOCs emitted by T. atroviride LZ42 on the mycelial growth of the plant pathogen, a volatile antifungal assay was conducted. We found that the colony diameters of the F. oxysporum treated with the VOCs of T. atroviride LZ42 were smaller than those of the control (Figure 4). The percentage inhibition of F. oxysporum by the volatiles from T. atroviride LZ42 was $54.57 \%$.

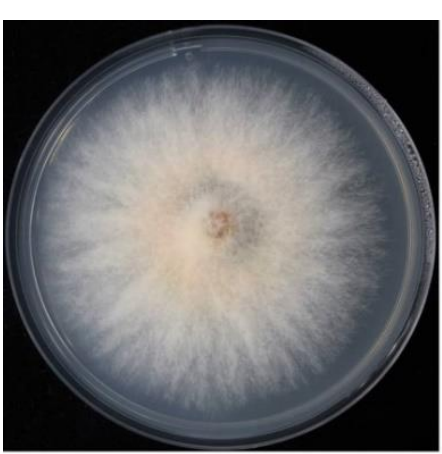

F. oxysporum

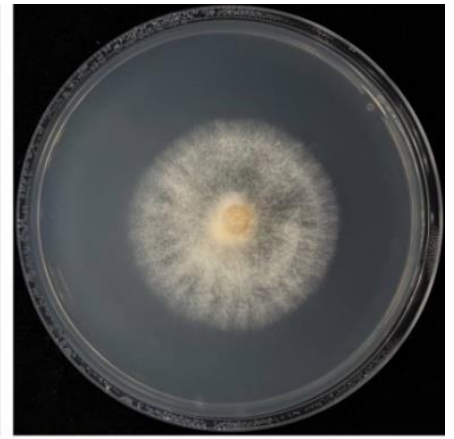

F. oxysporum + VOCs

Figure 4. Antifungal activity of the volatile organic compounds emitted by Trichoderma atroviride LZ42 on the mycelial growth of Fusarium oxysporum f. sp. lycopersici $\mathrm{K} 34$. 
Based on the SPME GC/MS analysis and comparison of the mass spectrum in the GC-MS system data bank, the VOCs produced by T. atroviride LZ42 on PDA medium were tentatively identified as five compounds based on similarities greater than $90 \%$ : 2-heptanone, 2-pentyl furan (2-PF), $\beta$-phellandrene, 3,5,5-trimethyl-cyclohexene, and 6-pentyl-2H-pyran-2-one (6-PP) (Table 2). The data from the relative area of each peak showed that 6-pentyl-2H-pyran-2-one was the most abundant compound emitted by $T$. atroviride LZ42, followed by 2-pentyl furan and $\beta$-phellandrene. Figure 5 shows the mass spectrum of the five compounds produced by T. atroviride LZ42.

Table 2. International Union of Pure and Applied Chemistry (IUPAC) names of the volatile compounds produced by Trichoderma atroviride LZ42 identified through SPME GC/MS analysis.

\begin{tabular}{cccccc}
\hline RT (min) & Volatile compound & $\mathbf{m} / \mathbf{z}$ & Formula & Similarity & \% Area \\
\hline $\mathbf{1 2 . 2 5}$ & 2-heptanone & 114 & $\mathrm{C}_{7} \mathrm{H}_{14} \mathrm{O}$ & 92 & 2.74 \\
$\mathbf{1 3 . 1 3}$ & 2-pentylfuran & 138 & $\mathrm{C}_{9} \mathrm{H}_{14} \mathrm{O}$ & 93 & 3.54 \\
$\mathbf{1 4 . 2 8}$ & $\beta$-phellandrene & 136 & $\mathrm{C}_{10} \mathrm{H}_{16}$ & 89 & 3.02 \\
$\mathbf{1 8 . 3 9}$ & 3,5,5-trimethyl-cyclohexene & 124 & $\mathrm{C}_{9} \mathrm{H}_{16}$ & 90 & 2.28 \\
$\mathbf{2 8 . 1 4}$ & 6-pentyl-2H-pyran-2-one & 166 & $\mathrm{C}_{10} \mathrm{H}_{14} \mathrm{O}_{2}$ & 94 & 48.26 \\
\hline
\end{tabular}

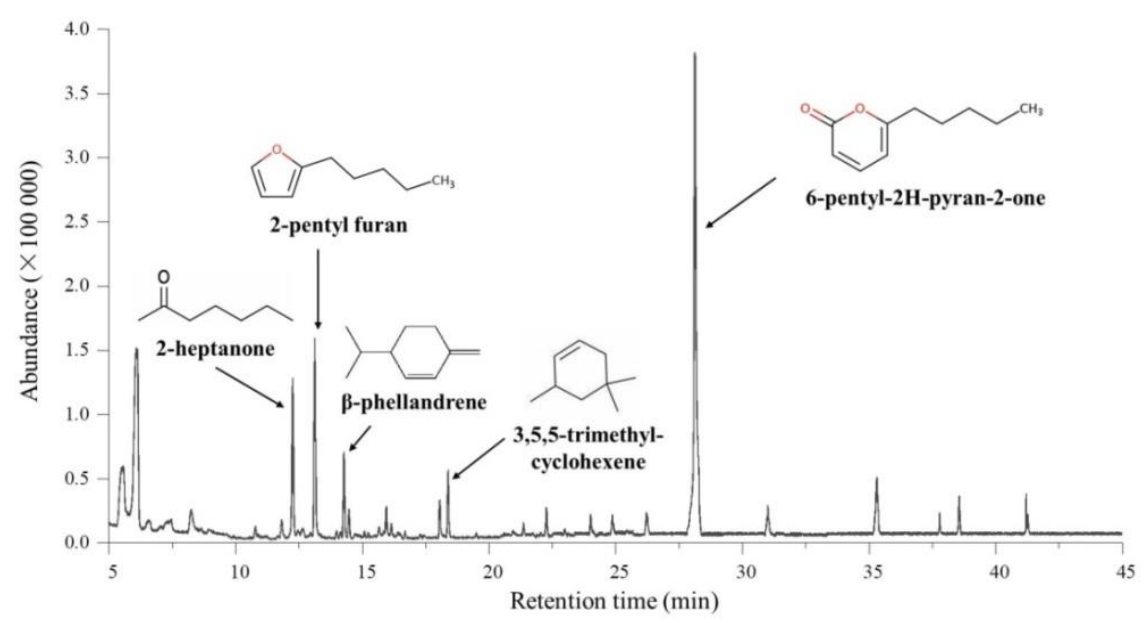

Figure 5. Chromatogram of the volatiles emitted by Trichoderma atroviride LZ42 analyzed through SPME GC/MS.

\subsection{Effects of pure VOCs on root growth of tomato seedlings}

The effects of the five selected volatiles on the directional growth and growth rate of tomato seedling roots were tested in Y-tube olfactometers. Among these compounds, only 6-PP exhibited a strong ability to influence the direction of primary roots (Figure $6 \mathrm{~A}$; Obs.Freq $=0.80 ; \mathrm{P}=0.10$ ). No significant differences in the treatments with 2-heptanone, 2-PF, $\beta$-phellandrene, and 3,5,5-trimethyl-cyclohexene were found. Intriguingly, there were no differences in the length of the primary roots between the treatments with any of the tested compounds and the control (Figure 6B). Among these five compounds, only 6-PP significantly increased the total root dry weight of tomatoes (Figure 6C). 


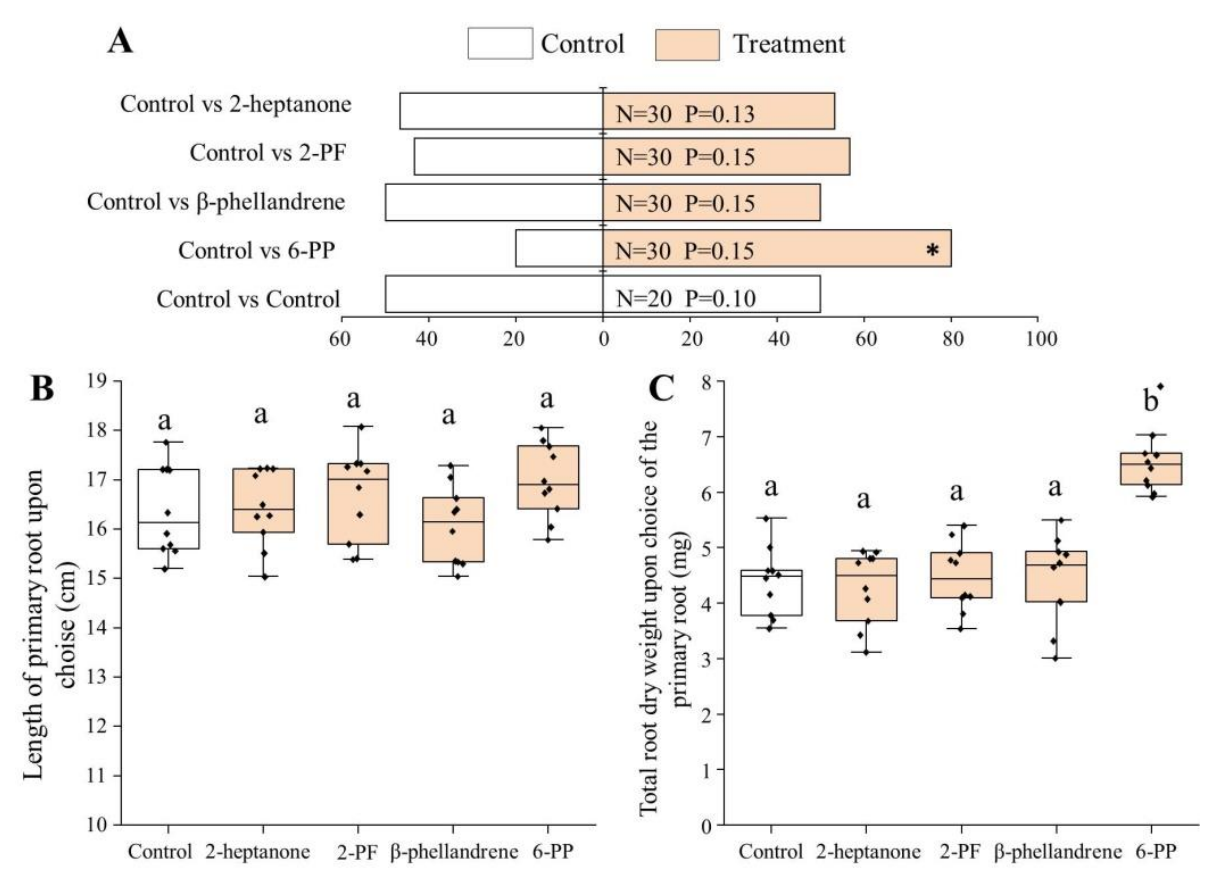

Figure 6. Effects of pure volatile compounds on the root growth of tomato seedlings using modified root Y-tube olfactometers. (A) Percentages of tomato primary roots growing in the control arm or the treatment arm 14 days after sowing. Control versus control was used as a negative control. $\mathrm{N}$, the number of replicates. Asterisks $\left(^{*}\right)$, pairwise differences between control and treatment choices $\left(\mathrm{H}_{0}=0.50\right)$. (B) Length of the primary root. (C) Total root dry weight. Different letters indicate significant differences derived from Duncan's multiple range test of one-way ANOVA at $\mathrm{P}=0.05$.

\subsection{Effects of pure VOCs on antifungal activity}

The selected pure VOCs were tested for antagonistic activity against the pathogen $F$. oxysporum on PDA plates. 6-PP produced the strongest inhibitory effect on mycelial growth (Table 3). The EC50 of 6-PP against F. oxysporum was $5.76 \mu \mathrm{L} \mathrm{mL}^{-1}$ headspace, which is significantly lower than the $18.72 \mu \mathrm{L} \mathrm{mL}^{-1}$ headspace for 2-PF and $34.77 \mu \mathrm{L}$ $\mathrm{mL}^{-1}$ headspace for 2-heptanone.

Table 3. Inhibitory effects of volatile compounds on mycelial growth of Fusarium oxysporum at $72 \mathrm{~h}$ post-inoculation.

\begin{tabular}{|c|c|}
\hline Chemicals & $\mathrm{EC}_{50}\left(\mu \mathrm{L} \mathrm{mL} \mathrm{m}^{-1}\right.$ headspace$)$ \\
\hline 2-heptanone & 34.77 \\
\hline 2-pentylfuran & 18.72 \\
\hline$\beta$-phellandrene & - \\
\hline 3,5,5-trimethyl-cyclohexene & - \\
\hline 6-pentyl-2H-pyran-2-one & 5.76 \\
\hline
\end{tabular}

\section{Discussion}

Plant biostimulants formulated with beneficial microorganisms have been developed and applied for the purposes of promoting plant growth, development, and adaptation to abiotic or biotic stress [3]. Among them, Trichoderma-based biostimulants have received much attention because of their capacity to control plant diseases caused by certain fungi [34]. Thereafter, Trichoderma spp. have been widely studied and are among the fungi most commonly used in agriculture, horticulture, and forestry $[2,4,35]$. However, there is still considerable interest in finding new isolates of Trichoderma with more efficient biocontrol or plant biostimulatory activity for the development of more efficient plant biostimulants [36]. In this study, we identified and investigated a coconut-scented 
T. atroviride strain LZ42 with a plant-growth-promoting effect and biocontrol activity. $T$. atroviride LZ42 formulated in talc exhibited a significant control effect on Fusarium wilt of tomatoes in greenhouses and promoted the accumulation of plant biomass and growth, which is consistent with the activity of another T. atroviride strain BC0584 [37]. The greenhouse experiments in this study clearly showed the positive effect of T. atroviride LZ42 on Fusarium wilt disease control. Our results suggest that T. atroviride LZ42 has potential as a biostimulant and/or a biocontrol agent for application during vegetable or crop growth.

The mechanism through which Trichoderma results in biostimulation involves multilevel communication with the root systems of the host plant [38]. Many Trichoderma strains are endophytic plant opportunistic symbionts that colonize the root, leading to the activation of defense and developmental responses of the host plant [39-41]. The secondary metabolites released into the rhizosphere by Trichoderma strains, including auxins, small peptides, and other active metabolites, can promote root branching and nutrient-uptake capacity, thereby boosting plant growth and yield [11]. T. atroviride LZ42 exerted a growth-promoting effect and enhanced root development in the tomato seedlings in this study. This might be associated with the production of indole acetic acid (IAA) and 1-aminocyclopropane-1-carboxylate (ACC) deaminase by $T$. atroviride $[20,42]$. However, we focused on the effect of VOCs emitted by T. atroviride LZ42 on the root growth of tomato seedlings using a root Y-tube olfactometer assay in this study. We found that the primary roots of tomato seedlings grew more frequently toward volatiles from T. atroviride LZ42 than the control. The root Y-tube olfactometer method had been successfully developed and used to investigate the chemical crosstalk between tomato roots and soil-borne fungi and its impact on root growth [31]. To the best of our knowledge, this is the first study to characterize the effects of VOCs produced by T. atroviride on the plant root growth in Y-tube olfactometers.

Fungi produce various mixtures of VOCs that are able to diffuse through the atmosphere and soils [18]. Fungal VOCs are considered important signals for fungi in their natural environments [43]. Fungi use their VOCs to interact with plants, arthropods, bacteria, and other fungi in various habitats [44]. In particular, the roles of VOCs produced by Trichoderma spp. in stimulating plant growth and suppressing the growth of plant pathogens have been proven [20]. Our results demonstrate the ability of T. atroviride LZ42 to produce volatile antifungal compounds, showing that this is an important mechanism involved in and responsible for the successful inhibition of F. oxysporum and effective control of Fusarium wilt disease in tomato seedlings. The VOCs produced by $\mathrm{T}$. atroviride LZ42 positively directed the growth of the primary roots of tomato seedlings and increased the biomass of the roots. These results are consistent with those for other T. atroviride strains such as IMI 206040 [25] and CBS 01-209 [21].

These volatiles produced by fungi have potential for agricultural application, motivating the search for additional bioactive VOCs from a variety of Trichoderma species [45]. Among these VOCs, 6-PP, a polyketide with a characteristic sweet coconut-like aroma, is the most common and well-known compound produced by various Trichoderma species [45]. 6-PP is regarded as a potential volatile chemical with a broad-spectrum ability to modulate both root growth and defense responses [21]. Our results show that 6-PP is the dominant VOC released by T. atroviride LZ42 after identification through SPME GC/MS, in agreement with the strong coconut-like scent from the host fungus. The results of the current study demonstrate that 6-PP emitted by T. atroviride LZ42 is the critical factor in fungal biostimulation and biocontrol. Various evidences from the literatures support this result. Taha et al. (2021) indicated that 6-PP elicited the induction of SAR in tobacco and exhibited antiviral activity against tobacco mosaic virus [46]. Garnica-Vergara et al. (2016) found that 6-PP improved the shoot and root growth and total biomass production of Arabidopsis thaliana [25]. 6-PP was also shown to control several plant pathogenic fungi such as F. moniliforme [47], Rhizoctonia solani [48], and Athelia rolfsii [49]. Pascale et al. (2017) suggested that using natural compounds such as 6-PP produced by Trichoderma spp. may be an effective alternative strategy [50]. 
Manipulating the production of bioactive VOCs to improve the performance of Trichoderma products remains a challenge. The production of bioactive VOCs in Trichoderma spp. is considered to be strain- and condition-dependent [34,51]. Flores et al. (2019) reported that 6-PP production by $T$. atroviride was mainly elicited by the metabolites from the plant pathogenic fungus Rhizoctonia solani [52]. However, Lee et al. (2016) found that 6-PP was not common to all Trichoderma strains [21]. It is surprising that 6-PP does not exist in the VOC profile of either T. virens strain G-41 or T. harzianum strain T-22 [24]. Though the VOCs produced by Trichoderma are much more complex than previously considered, their chemical diversity and communication roles in plant-fungus cross-kingdom signaling are still of interest.

In summary, we identified the plant biostimulatory and biocontrol activity of $T$. atroviride strain LZ42 on tomato seedlings, which were manifested in plant root growth stimulation and the suppression of disease symptoms. The VOCs produced by T. atroviride LZ42, especially 6-PP, are involved in its biostimulatory and biocontrol activities. The results from our study reveal that VOCs emitted by T. atroviride LZ42 mediate directed root growth, improve plant growth, and suppress Fusarium wilt disease in tomato plants.

Supplementary Materials: The following are available online at www.mdpi.com/xxx/s1, Table S1: GenBank accession numbers of taxa used in phylogenetic analyses..

Author Contributions: Conceptualization, Y.W.; methodology, Y.W.; software, H.J.; validation, Y.R., L.Z. and H.J.; formal analysis, Y.W.; investigation, H.J.; resources, Y.W.; data curation, H.J.; writing - original draft preparation, H.J.; writing - review and editing, Y.W.; visualization, H.J.; supervision, Y.W.; project administration, Y.W.; funding acquisition, Y.W. All authors have read and agreed to the published version of the manuscript.

Funding: This research was funded by a grant from the Zhejiang Key Research and Development Program of China (2019C0203002) and a grant from National Key Research and Development Program of China (2017YFD0201100-7).

Institutional Review Board Statement: Not applicable.

Acknowledgments: The authors give very special thanks to Dr. Bin Wang (Zhejiang A \& F University, China) for his assistance in the greenhouse and MDPI (https://www.mdpi.com) for English language editing.

Conflicts of Interest: The authors declare no conflict of interest.

\section{References}

1. Schuster, A.; Schmoll, M. Biology and biotechnology of Trichoderma. Appl. Microbiol. Biotechnol. 2010, 87, 787-799, doi:10.1007/s00253-010-2632-1.

2. López-Bucio, J.; Pelagio-Flores, R.; Herrera-Estrella, A. Trichoderma as biostimulant: exploiting the multilevel properties of a plant beneficial fungus. Sci. Hortic. 2015, 196, 109-123, doi:10.1016/J.SCIENTA.2015.08.043.

3. Woo, S.L.; Ruocco, M.; Vinale, F.; Nigro, M.; Marra, R.; Lombardi, N.; Pascale, A.; Lanzuise, S.; Manganiello, G.; Lorito, M. Trichoderma-based products and their widespread use in agriculture. Open Mycol. J. 2014, 8, 71-126, doi:10.2174/1874437001408010071.

4. Zin, N.A.; Badaluddin, N.A. Biological functions of Trichoderma spp. for agriculture applications. Ann. Agric. Sci. 2020, 65, 168-178, doi:10.1016/j.aoas.2020.09.003.

5. Harman, G.E.; Howell, C.R.; Viterbo, A.; Chet, I.; Lorito, M. Trichoderma species--opportunistic, avirulent plant symbionts. Nat. Rev. Microbiol. 2004, 2, 43-56, doi:10.1038/nrmicro797.

6. Sallam, N.M.A.; Eraky, A.M.I.; Sallam, A. Effect of Trichoderma spp. on Fusarium wilt disease of tomato. Mol. Biol. Rep. 2019, 46, 4463-4470, doi:10.1007/s11033-019-04901-9. 
7. Zhou, C.; Guo, R.; Ji, S.; Fan, H.; Wang, J.; Wang, Y.; Liu, Z. Isolation of Trichoderma from forestry model base and the antifungal properties of isolate TpsT17 toward Fusarium oxysporum. Microbiol. Res. 2020, 231, 126371, doi:10.1016/j.micres.2019.126371.

8. Nath, V.S.; John, N.S.; Anjanadevi, I.P.; Hegde, V.M.; Jeeva, M.L.; Misra, R.S.; Veena, S.S. Characterization of Trichoderma spp. antagonistic to Phytophthora colocasiae associated with leaf blight of taro. Ann. Microbiol. 2014, 64, 1513-1522, doi:10.1007/s13213-013-0794-7.

9. Guo, Y.; Fan, Z.; Yi, X.; Zhang, Y.; Khan, R.A.A.; Zhou, Z. Sustainable management of soil-borne bacterium Ralstonia solanacearum in vitro and in vivo through fungal metabolites of different Trichoderma spp. Sustainability 2021, 13, 1491, doi:10.3390/SU13031491.

10. Khan, R.A.A.; Najeeb, S.; Mao, Z.; Ling, J.; Yang, Y.; Li, Y.; Xie, B. Bioactive secondary metabolites from Trichoderma spp. against phytopathogenic bacteria and root-knot nematode. Microorganisms 2020, 8, 401, doi:10.3390/microorganisms8030401.

11. Contreras-Cornejo, H.A.; Macías-Rodríguez, L.; del-Val, E.; Larsen, J. Ecological functions of Trichoderma spp. and their secondary metabolites in the rhizosphere: interactions with plants. FEMS Microbiol. Ecol. 2016, 92, fiw036, doi:10.1093/FEMSEC/FIW036.

12. Esparza-Reynoso, S.; Ruíz-Herrera, L.F.; Pelagio-Flores, R.; Macías-Rodríguez, L.I.; Martínez-Trujillo, M.; López-Coria, M.; Sánchez-Nieto, S.; Herrera-Estrella, A.; López-Bucio, J. Trichoderma atroviride-emitted volatiles improve growth of Arabidopsis seedlings through modulation of sucrose transport and metabolism. Plant, Cell Environ. 2021, 44, 1961-1976, doi:10.1111/PCE.14014.

13. Guzman-Guzman, P.; Porras-Troncoso, M.D.; Olmedo-Monfil, V.; Herrera-Estrella, A. Trichoderma Species: Versatile Plant Symbionts. Phytopathology 2019, 109, 6-16, doi:10.1094/PHYTO-07-18-0218-RVW.

14. Macías-Rodríguez, L.; Guzmán-Gómez, A.; García-Juárez, P.; Contreras-Cornejo, H.A. Trichoderma atroviride promotes tomato development and alters the root exudation of carbohydrates, which stimulates fungal growth and the biocontrol of the phytopathogen Phytophthora cinnamomi in a tripartite interaction system. FEMS Microbiol. Ecol. 2018, 94, fiy137, doi:10.1093/femsec/fiy137.

15. Kottb, M.; Gigolashvili, T.; Großkinsky, D.K.; Piechulla, B. Trichoderma volatiles effecting Arabidopsis: from inhibition to protection against phytopathogenic fungi. Front. Microbiol. 2015, 6, 995, doi:10.3389/fmicb.2015.00995.

16. Ojaghian, S.; Wang, L.; Xie, G.; Zhang, J. Effect of volatiles produced by Trichoderma spp. on expression of glutathione transferase genes in Sclerotinia sclerotiorum. Biol. Control 2019, 136, 103999, doi:10.1016/j.biocontrol.2019.103999.

17. Wonglom, P.; Ito, S.; Sunpapao, A. Volatile organic compounds emitted from endophytic fungus Trichoderma asperellum T1 mediate antifungal activity, defense response and promote plant growth in lettuce (Lactuca sativa). Fungal Ecol. 2020, 43, 100867, doi:10.1016/j.funeco.2019.100867.

18. Guo, Y.; Jud, W.; Ghirardo, A.; Antritter, F.; Benz, J.P.; Schnitzler, J.P.; Rosenkranz, M. Sniffing fungi - phenotyping of volatile chemical diversity in Trichoderma species. New Phytol. 2020, 227, 244-259, doi:10.1111/nph.16530.

19. Lemfack, M.C.; Gohlke, B.O.; Toguem, S.M.T.; Preissner, S.; Piechulla, B.; Preissner, R. mVOC 2.0: a database of microbial volatiles. Nucleic Acids Res. 2018, 46, D1261-D1265, doi:10.1093/nar/gkx1016.

20. Contreras-Cornejo, H.A.; López-Bucio, J.S.; Méndez-Bravo, A.; Macías-Rodríguez, L.I.; Ramos-Vega, M.; Guevara-García, A.; López-Bucio, J. Trichoderma atroviride alters Arabidopsis root-system architecture modulating the mitogenactivated protein kinase 6 activity through ethylene and auxin signaling pathways. Mol. Plant-Microbe Interact. 2015, 28, 701-710, doi:10.1094/MPMI-01-15-0005-R.

21. Lee, S.; Yap, M.; Behringer, G.; Hung, R.; Bennett, J.W. Volatile organic compounds emitted by Trichoderma species mediate plant growth. Fungal Biol. Biotechn. 2016, 3, 7, doi:10.1186/s40694-016-0025-7. 
22. Nieto-Jacobo, M.F.; Steyaert, J.M.; Salazar-Badillo, F.B.; Nguyen, D.V.; Rostas, M.; Braithwaite, M.; De Souza, J.T.; Jimenez-Bremont, J.F.; Ohkura, M.; Stewart, A.; et al. Environmental growth conditions of Trichoderma spp. affects indole acetic acid derivatives, volatile organic compounds, and plant growth promotion. Front. Plant Sci. 2017, 8, 102, doi:10.3389/fpls.2017.00102.

23. Guo, Y.; Ghirardo, A.; Weber, B.; Schnitzler, J.-P.; Benz, J.P.; Rosenkranz, M. Trichoderma species differ in their volatile profiles and in antagonism toward Ectomycorrhiza laccaria bicolor. Front. Microbiol. 2019, 10, 891, doi:10.3389/FMICB.2019.00891.

24. Li, N.; Alfiky, A.; Wang, W.; Islam, M.; Nourollahi, K.; Liu, X.; Kang, S. Volatile compound-mediated recognition and inhibition between Trichoderma biocontrol agents and Fusarium oxysporum. Front. Microbiol. 2018, 9, 2614, doi:10.3389/fmicb.2018.02614.

25. Garnica-Vergara, A.; Barrera-Ortiz, S.; Munoz-Parra, E.; Raya-Gonzalez, J.; Mendez-Bravo, A.; Macias-Rodriguez, L.; Ruiz-Herrera, L.F.; Lopez-Bucio, J. The volatile 6-pentyl-2H-pyran-2-one from Trichoderma atroviride regulates Arabidopsis thaliana root morphogenesis via auxin signaling and ETHYLENE INSENSITIVE 2 functioning. New Phytol. 2016, 209, 1496-1512, doi:10.1111/nph.13725.

26. Tong, X.; Jiang, H.; Liang, Y.; Rao, Y.; Mei, L.; Wang, Y. Waterlogging reduces soil colonization by antagonistic fungi and restores production in Ganoderma lucidum continuous cultivation. Crop Protect. 2020, 137, 105314, doi:10.1016/j.cropro.2020.105314.

27. Liu, Y.J.; Whelen, S.; Hall, B.D. Phylogenetic relationships among ascomycetes: evidence from an RNA polymerse II subunit. Mol. Biol. Evol. 1999, 16, 1799-1808, doi:10.1093/oxfordjournals.molbev.a026092.

28. Carbone, I.; Kohn, L.M. A method for designing primer sets for speciation studies in filamentous ascomycetes. Mycologia 1999, 91, 553-556, doi:10.2307/3761358.

29. Kumar, S.; Stecher, G.; Tamura, K. MEGA7: Molecular Evolutionary Genetics Analysis version 7.0 for bigger datasets. Mol. Biol. Evol. 2016, 33, 1870-1870, doi:10.1093/molbev/msw054.

30. Mei, L.; Liang, Y.; Zhang, L.; Wang, Y.; Guo, Y. Induced systemic resistance and growth promotion in tomato by an indole-3-acetic acid-producing strain of Paenibacillus polymyxa. Ann. Appl. Biol. 2014, 165, 270-279, doi:10.1111/AAB.12135.

31. Moisan, K.; Raaijmakers, J.M.; Dicke, M.; Lucas-Barbosa, D.; Cordovez, V. Volatiles from soil-borne fungi affect directional growth of roots. Plant, Cell Environ. 2021, 44, 339-345, doi:10.1111/pce.13890.

32. Stoppacher, N.; Kluger, B.; Zeilinger, S.; Krska, R.; Schuhmacher, R. Identification and profiling of volatile metabolites of the biocontrol fungus Trichoderma atroviride by HS-SPME-GC-MS. J. Microbiol. Methods 2010, 81, 187-193, doi:10.1016/j.mimet.2010.03.011.

33. Wu, F.; Tong, X.; Zhang, L.; Mei, L.; Guo, Y.; Wang, Y. Suppression of Rhizopus fruit rot by volatile organic compounds produced by Paenibacillus polymyxa CF05. Biocontrol Sci. Technol. 2020, 30, 1351-1364, doi:10.1080/09583157.2020.1826902.

34. Vinale, F.; Sivasithamparam, K.; Ghisalberti, E.L.; Marra, R.; Barbetti, M.J.; Li, H.; Woo, S.L.; Lorito, M. A novel role for Trichoderma secondary metabolites in the interactions with plants. Physiol. Mol. Plant Pathol. 2008, 72, 80-86, doi:10.1016/j.pmpp.2008.05.005.

35. Ruiz-Gómez, F.J.; Miguel-Rojas, C. Antagonistic potential of native Trichoderma spp. against Phytophthora cinnamomi in the control of holm oak decline in Dehesas ecosystems. Forests 2021, 12, 945, doi:10.3390/f12070945.

36. Sood, M.; Kapoor, D.; Kumar, V.; Sheteiwy, M.S.; Ramakrishnan, M.; Landi, M.; Araniti, F.; Sharma, A. Trichoderma: The "secrets" of a multitalented biocontrol agent. Plants 2020, 9, doi:10.3390/plants9060762.

37. Coninck, E.; Scauflaire, J.; Gollier, M.; Lienard, C.; Foucart, G.; Manssens, G.; Munaut, F.; Legreve, A. Trichoderma atroviride as a promising biocontrol agent in seed coating for reducing Fusarium damping-off on maize. J. Appl. Microbiol. 2020, 129, 637-651, doi:10.1111/jam.14641. 
38. Alfiky, A.; Weisskopf, L. Deciphering Trichoderma-plant-pathogen interactions for better development of biocontrol applications. J Fungi (Basel) 2021, 7, 61, doi:10.3390/jof7010061.

39. Brotman, Y.; Landau, U.; Cuadros-Inostroza, A.; Tohge, T.; Fernie, A.R.; Chet, I.; Viterbo, A.; Willmitzer, L. Trichoderma-plant root colonization: escaping early plant defense responses and activation of the antioxidant machinery for saline stress tolerance. PLoS Pathog. 2013, 9, e1003221, doi:10.1371/journal.ppat.1003221.

40. Kakabouki, I.; Tataridas, A.; Mavroeidis, A.; Kousta, A.; Karydogianni, S.; Zisi, C.; Kouneli, V.; Konstantinou, A.; Folina, A.; Konstantas, A.; et al. Effect of colonization of Trichoderma harzianum on growth development and CBD content of Hemp (Cannabis sativa L.). Microorganisms 2021, 9, 518, doi:10.3390/microorganisms9030518.

41. Salas-Marina, M.A.; Silva-Flores, M.A.; Uresti-Rivera, E.E.; Castro-Longoria, E.; Herrera-Estrella, A.; Casas-Flores, S. Colonization of Arabidopsis roots by Trichoderma atroviride promotes growth and enhances systemic disease resistance through jasmonic acid/ethylene and salicylic acid pathways. Eur. J. Plant Pathol. 2011, 131, 15-26, doi:10.1007/s10658-011-9782-6.

42. Estrada-Rivera, M.; Rebolledo-Prudencio, O.G.; Perez-Robles, D.A.; Rocha-Medina, M.D.C.; Gonzalez-Lopez, M.D.C.; Casas-Flores, S. Trichoderma histone deacetylase HDA-2 modulates multiple responses in Arabidopsis. Plant Physiol. 2019, 179, 1343-1361, doi:10.1104/pp.18.01092.

43. Li, N.; Alfiky, A.; Vaughan, M.M.; Kang, S. Stop and smell the fungi: Fungal volatile metabolites are overlooked signals involved in fungal interaction with plants. Fungal Biol. Rev. 2016, 30, 134-144, doi:10.1016/J.FBR.2016.06.004.

44. Morath, S.U.; Hung, R.; Bennett, J.W. Fungal volatile organic compounds: A review with emphasis on their biotechnological potential. Fungal Biol. Rev. 2012, 26, 73-83, doi:10.1016/J.FBR.2012.07.001.

45. Jeleń, H.; Błaszczyk, L.; Chełkowski, J.; Rogowicz, K.; Strakowska, J. Formation of 6-n-pentyl-2H-pyran-2-one (6-PAP) and other volatiles by different Trichoderma species. Mycol. Prog. 2013, 13, 589-600, doi:10.1007/S11557-013-0942-2.

46. Taha, M.A.; Ismaiel, A.A.; Ahmed, R.M. 6-pentyl- $\alpha$-pyrone from Trichoderma koningii induces systemic resistance in tobacco against tobacco mosaic virus. Eur. J. Plant Pathol. 2020, 159, 81-93, doi:10.1007/s10658-020-02142-2.

47. El-Hasan, A.; Buchenauer, H. Actions of 6-pentyl-alpha-pyrone in controlling seedling blight incited by Fusarium moniliforme and inducing defence responses in maize. J. Phytopathol. 2009, 157, 697-707, doi:10.1111/J.1439-0434.2009.01565.X.

48. Worasatit, N.; Sivasithamparam, K.; Ghisalberti, E.L.; Rowland, C. Variation in pyrone production, lytic enzymes and control of rhizoctonia root rot of wheat among single-spore isolates of Trichoderma koningii. Mycol. Res. 1994, 98, 1357-1363, doi:10.1016/s0953-7562(09)81063-0.

49. Dodd, S.L.; Hill, R.A.; Stewart, A. Control of Athelia rolfsii disease on lentil seedlings using 6-pentyl- $\alpha$-pyrone. Soil Biol. Biochem. 2000, 32, 1033-1034, doi:10.1016/s0038-0717(00)00003-1.

50. Pascale, A.; Vinale, F.; Manganiello, G.; Nigro, M.; Lanzuise, S.; Ruocco, M.; Marra, R.; Lombardi, N.; Woo, S.L.; Lorito, M. Trichoderma and its secondary metabolites improve yield and quality of grapes. Crop Protect. 2017, 92, 176-181, doi:10.1016/j.cropro.2016.11.010.

51. Lee, S.; Hung, R.; Yap, M.; Bennett, J.W. Age matters: the effects of volatile organic compounds emitted by Trichoderma atroviride on plant growth. Arch. Microbiol. 2015, 197, 723-727, doi:10.1007/s00203-015-1104-5.

52. Flores, C.; Nieto, M.; Millán-Gómez, D.V.; Caro, M.; Galindo, E.; Serrano-Carreón, L. Elicitation and biotransformation of 6-pentyl- $\alpha$-pyrone in Trichoderma atroviride cultures. Process Biochem. 2019, 82, 68-74, doi:10.1016/J.PROCBIO.2019.04.019. 